\title{
Terapia audiovisual. Propuesta para disminuir ansiedad en pacientes quemados durante su estancia en la unidad de cuidados intensivos
}

Audiovisual therapy. Proposal to decrease anxiety in burned patients during their stay in the Intensive Care Unit Terapia audiovisual. Proposta para reduzir a ansiedade em pacientes queimados durante sua estadia em Unidade de Terapia Intensiva

Marco Antonio Garnica Escamilla, ${ }^{*}$ Roxana Morales Cruz, ${ }^{\ddagger}$ Nanhiely Rodríguez Ochoa, ${ }^{*}$ Eunice Sofía Vargas Torres, ${ }^{*}$ Oscar Miguel Marín Landa*

\section{RESUMEN}

La atención y manejo del paciente quemado en México representa un reto para el personal médico, la estancia intrahospitalaria del quemado generalmente es prolongada, por lo que los pacientes pueden desarrollar complicaciones psicológicas derivadas del cambio en su aspecto físico y los tratamientos que este tipo de patología requiere, por lo que la aparición de ansiedad es común. El propósito de este trabajo es plantear una propuesta de terapia audiovisual como tratamiento alternativo y/o complementario en el control de la ansiedad en el paciente durante su estancia en la unidad de cuidados intensivos.

Palabras clave: Paciente quemado, rehabilitación, dolor, ansiedad, terapia audiovisual.

\section{ABSTRACT}

The care and management of the burn patient in Mexico represents a challenge for the medical staff, the hospital stay of the burn is generally prolonged so that patients can develop psychological complications derived from the change in their physical appearance and the treatments that this type of pathology requires, so the appearance of anxiety is common. The purpose of this work is to propose a proposal of audiovisual therapy as an alternative and/or complementary treatment in the control of anxiety in the patient during his stay in the intensive care unit.

Keywords: Burned patient, rehabilitation, pain, anxiety, audiovisual therapy.

\section{RESUMO}

O cuidado e o manejo do paciente queimado no México representam um desafio para a equipe médica, a internação do paciente queimado é geralmente prolongada para que os pacientes possam desenvolver complicações psicológicas derivadas da mudança em sua aparência física e dos tratamentos que este tipo de a patologia exige, então o aparecimento de ansiedade é comum. O objetivo deste trabalho é propor uma proposta de terapia audiovisual como alternativa e/ou tratamento complementar no controle da ansiedade do paciente durante sua permanência em unidade de terapia intensiva.

Palavras-chave: Paciente queimado, reabilitação, dor, ansiedade, terapia audiovisual.

\section{INTRODUCCIÓN}

El paciente gran quemado constituye un gran reto en el tratamiento para los profesionales sanitarios por las implicaciones orgánicas y biopsicosociales que requiere, por lo tanto en la atención de los pacientes debe participar un equipo multidisciplinario para asegurar una recuperación lo más exitosa posible, dicho equipo debe

\footnotetext{
* Centro Nacional de Investigación y Atención de Quemados. Instituto Nacional de Rehabilitación Luis Guillermo Ibarra Ibarra. CDMX, México.

₹ Centro Médico Naval. CDMX, México.

Recibido: 27/01/2020. Aceptado: 22/03/2020

Citar como: Garnica EMA, Morales CR, Rodríguez ON, Vargas TES, Marín LOM. Terapia audiovisual. Propuesta para disminuir ansiedad en pacientes quemados durante su estancia en la unidad de cuidados intensivos. Med Crit. 2021;35(2): 96-100. https:// dx.doi.org/10.35366/99530

www.medigraphic.com/medicinacritica
}

incluir profesionales tales como médicos, enfermeras, rehabilitadores, psicólogos, trabajadores sociales, entre otros. ${ }^{1-4}$

Según estableció la Organización Mundial de la Salud (OMS) en 2008, las quemaduras ocuparon el quinto lugar como causa de mortalidad a nivel mundial y se estima que cada año ingresan en las unidades de quemados hasta 1,000 pacientes. $^{5}$

Los pacientes con enfermedades psicológicas previas tales como depresión y/o ansiedad tienen mayor riesgo de presentar una lesión por quemadura y además, una vez sufrida la lesión, pasan por estancias hospitalarias más prolongadas en comparación con pacientes $\sin$ antecedentes, ${ }^{6-10}$ por lo que se concluye que los factores psicosociales y los antecedentes psiquiátricos tienen un impacto negativo en la recuperación de los pacientes.

Los pacientes quemados presentan una elevada incidencia de problemas psicológicos derivados de la lesión, experimentan una transformación muy importante de su imagen corporal de forma aguda y traumática, sobre todo si las quemaduras afectan zonas que no se pueden ocultar o si han sufrido algún tipo de amputación. ${ }^{1,11}$ Durante su hospitalización viven de manera súbita un cambio especialmente complejo en sus patrones de vida que les coloca en una posición de mayor vulnerabilidad física y emocional con respecto a otros tipos de pacientes. ${ }^{12}$

El estrés postraumático derivado del miedo por la lesión presentada y los trastornos de ansiedad generados principalmente por el miedo anticipado a lo que les va a ocurrir en un futuro (desfiguración, cirugía reconstructiva, cambios en el rol familiar, laboral, etc.), así como otras circunstancias derivadas del ambiente hospitalario en el que se encuentran (el ruido generado por el equipo o personal a cargo, despertares nocturnos frecuentes, restricción de visitas, prohibición de hijos menores de edad a visitas), son problemas muy frecuentes durante la hospitalización del paciente quemado. ${ }^{13,14}$

Se han realizado varios estudios con diferentes técnicas alternativas de tecnología para disminuir la ansiedad en pacientes quemados, cada una de ellas con ventajas y desventajas. ${ }^{15,16}$ 
Este estudio tiene como finalidad dar a conocer una estrategia de terapia audiovisual con videos de lazos afectivos más significativos para el paciente implementada en la Unidad de Quemados del Centro Nacional de Investigación y Atención de Quemados en el Instituto Nacional de Rehabilitación Luis Guillermo Ibarra Ibarra (LGII) como alternativa para disminuir la ansiedad del paciente quemado durante su estancia intrahospitalaria.

\section{ANTECEDENTES}

Los trastornos de ansiedad forman el grupo de trastornos mentales más comunes en la población general, es un grupo de trastorno en el que el desarrollo de comorbilidades es muy frecuente, principalmente los trastornos depresivos. ${ }^{17,18}$

El manejo del paciente con trastorno de ansiedad es complejo, sobre todo si consideramos la dificultad del diagnóstico diferencial, la necesidad de una terapéutica específica y en ocasiones prolongada. ${ }^{19}$

La ansiedad en la unidad de terapia intensiva en pacientes quemados es una condición común, por lo que se debe tener particular atención en identificarla en alguna de estas dos presentaciones:

\section{Ansiedad normal}

La ansiedad es en principio una emoción humana de aparición normal, cuya función es emprender un estado de alerta mayor ante la presencia de una amenaza, generando una respuesta hiperadrenérgica y mayor tensión. ${ }^{20,21}$

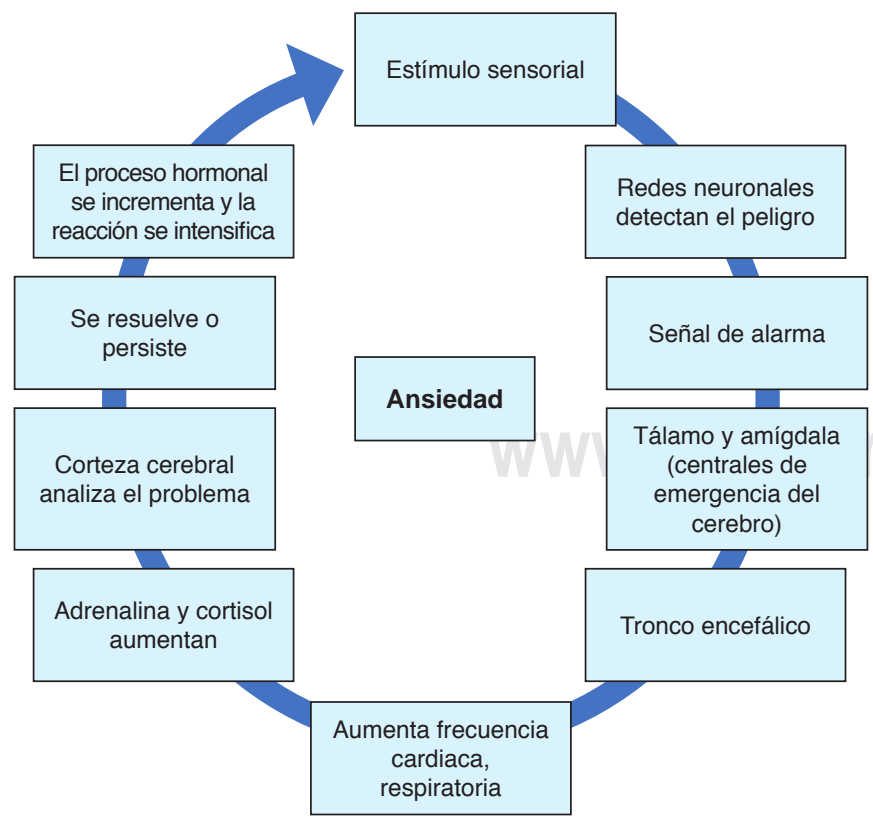

Figura 1: Fisiopatología de la ansiedad.

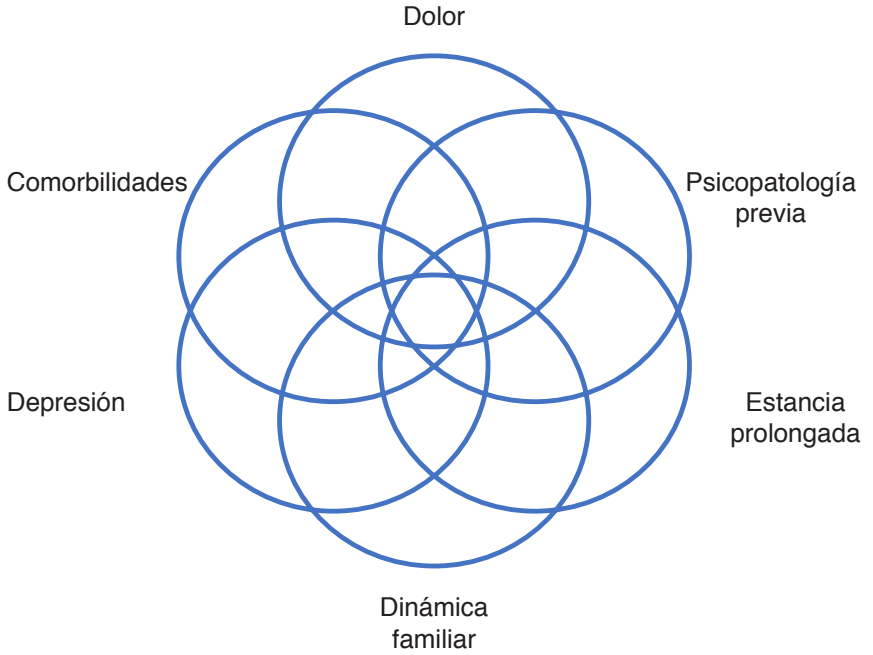

Figura 2: Factores desencadenantes de la ansiedad en el paciente quemado.

\section{Ansiedad patológica}

Cuando la ansiedad no es adaptativa, el peligro al que pretende responder no es real y el nivel de activación y su duración son desproporcionados con respecto a la situación objetiva, o bien cuando no es capaz de generar una respuesta adecuada o adaptativa, se habla de ansiedad patológica.

Por esta razón, la diferenciación entre lo que es normal y patológico se basa en la mayoría de los casos en criterios cuantitativos más o menos arbitrariamente consensuados, que de no controlarse generará un ataque de pánico (Figura 1).22

\section{FACTORES DESENCADENANTES}

Un aspecto que diferencia a la ansiedad del estrés, y también del miedo, es el peso tan importante que tiene en esta última respuesta el componente fisiológico. En cambio, tanto en la ansiedad como en el miedo el componente psicológico es el que resulta central. El estrés sólo puede convertirse en una condición patológica si las respuestas de adaptación del individuo se prologan en exceso en el tiempo y no son suficientes para hacer frente a los estresores, especialmente si se perciben como indicadores de amenaza o daño.

\section{Factores psicológicos}

En un paciente que ha sufrido quemaduras se puede llegar a desencadenar un trastorno de ansiedad debido a múltiples factores, entre los cuales se encuentran el miedo anticipado a lo que podrá pasar en un futuro inmediato, tal como la amputación de algún miembro 
del cuerpo, desfiguración, procedimientos quirúrgicos, el sonido de los aparatos médicos, cambios en el rol laboral y familiar, provocando malestar significativo con síntomas que afectan tanto en el aspecto físico como en el psicológico y/o conductual. ${ }^{23}$

\section{Factores ambientales}

Las causas de los trastornos de ansiedad no son totalmente conocidas, pero están implicados factores tanto biológicos como ambientales y psicosociales. Parece ser que es la interacción de múltiples determinantes lo que propicia la aparición de estos trastornos de ansiedad, siendo además muy común la comorbilidad con otros trastornos mentales como los trastornos del estado de ánimo (Figura 2).

El hecho de no poder incorporarse de forma inmediata a su trabajo para brindar el sustento a su familia es otro factor desencadenante de un posible trastorno de ansiedad.

O cuando se trata de la madre quien resulta afectada al no poder cuidar de sus hijos, abrazarlos y brindarles la protección necesaria.

E incluso al momento de incorporarse nuevamente a su hogar puede llegar a generar ansiedad por no poder realizar actividades de autocuidado, el tener que depender de algún familiar para llevar a cabo las actividades de la vida diaria, el temor de ser "señalado" o que se burlen de su aspecto físico es otro factor desencadenante de ansiedad.

\section{TRATAMIENTO}

\section{Farmacológico}

El tratamiento farmacológico de los diversos trastornos de ansiedad se basa en el empleo de diversos fármacos que modulan la respuesta exagerada que desarrolla el paciente. Entre los más utilizados están los inhibidores selectivos de la recaptura de serotonina y las benzodiacepinas (Figura 3).

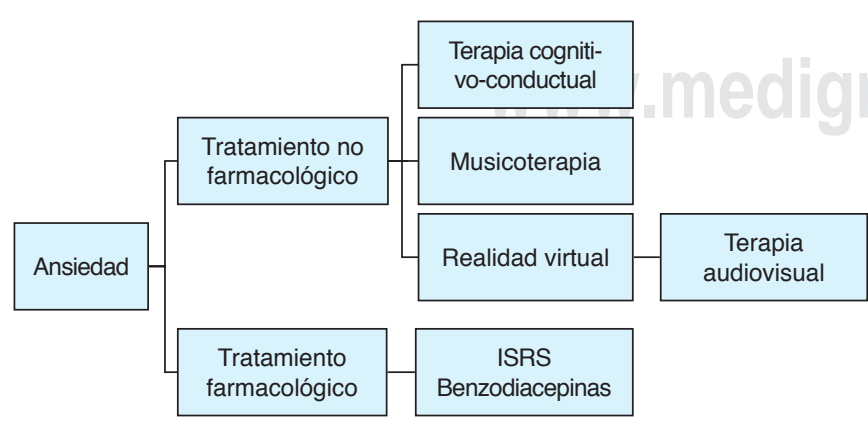

Figura 3: Tratamiento de la ansiedad. ISRS = inhibidores selectivos de la recaptura de serotonina.

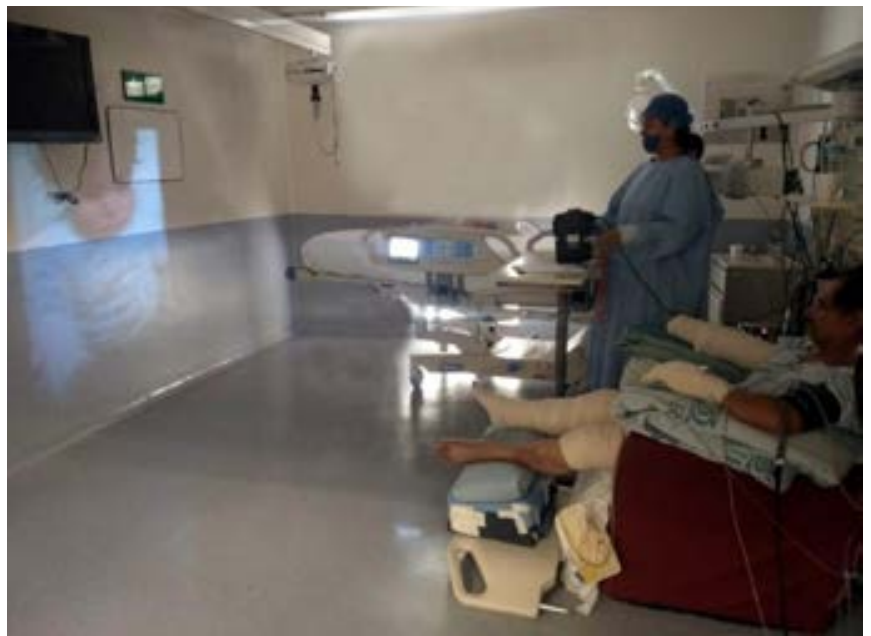

Figura 4: Proyección de material audiovisual de contenido familiar en la unidad de cuidados intensivos.

\section{Inhibidores de la recaptura de serotonina}

Utilizados de primera línea, excepto en los casos de ansiedad fóbica y agorafobia sin trastorno de pánico, en los que el tratamiento es fundamentalmente psicoterapéutico. ${ }^{24}$

\section{Benzodiacepinas}

Los demás trastornos de ansiedad generalmente mejoran con estos fármacos. Hoy en día, a pesar del lugar que ocupan las benzodiacepinas en el tratamiento de los síntomas de ansiedad, el papel principal ha sido otorgado a la familia de los denominados antidepresivos por su efecto ansiolítico derivado de sus acciones en los sistemas de serotonina y noradrenalina cerebrales. ${ }^{25}$

\section{No farmacológico}

La intervención psicológica en general debe considerarse como punto primordial en el tratamiento de este tipo de pacientes debido a que se ha demostrado ser incluso más resolutiva que algunas intervenciones psicofarmacológicas para estos trastornos.

En la actualidad es recomendable el uso de terapias no farmacológicas en conjunto con agentes farmacológicos para reducir la ansiedad durante el cuidado de las heridas del paciente quemado. ${ }^{26}$

Este tipo de abordaje psicoterapéutico se debe plantear desde varias concepciones teóricas.

\section{La terapia cognitivo-conductual}

Es un método activo y directivo en el que terapeuta y paciente trabajan en forma conjunta y estructurada con 
tareas fuera de sesión. Utiliza técnicas tanto conductuales como cognitivas y en combinación diferente según la sintomatología a abordar: relajación y respiración, entrenamiento autógeno, reestructuración cognitiva, exposición in vivo y diferida, detención del pensamiento, resolución de problemas. ${ }^{27-29}$

\section{Musicoterapia}

El abordaje terapéutico de la ansiedad a partir de la musicoterapia ha mostrado resultados favorables; sin embargo, aún no son suficientes. ${ }^{30}$

La musicoterapia tiene aplicabilidad durante el tratamiento del paciente quemado, este tipo de intervenciones inició a finales de 1970, siendo Christen Berry quien publicó el primer artículo aplicando la musicoterapia en pacientes quemados. ${ }^{31}$

\section{Realidad virtual (RV)}

La RV ha sido establecida como una nueva forma de distracción, otorgando al paciente la ilusión de inmersión en un entorno tridimensional generado por una computadora, la cual da al paciente la ilusión de estar físicamente en el lugar que en ese momento está observando. ${ }^{32}$

Este estudio propone la terapia audiovisual con diversos contenidos, ya sean de familiares (matrimonio, hijos, nietos, compadres, etc.), amigos, mascotas, grupos musicales y demás, como alternativa para disminuir la ansiedad del paciente quemado durante su estancia en la unidad de cuidados intensivos.

En el Centro Nacional de Investigación y Atención de Quemados se implementó una estrategia proyectando videos en la habitación de los pacientes, los cuales eran solicitados por el personal médico o psicólogos a familiares y que eran grabados utilizando las cámaras de sus teléfonos celulares (Figuras 4 y 5 ).

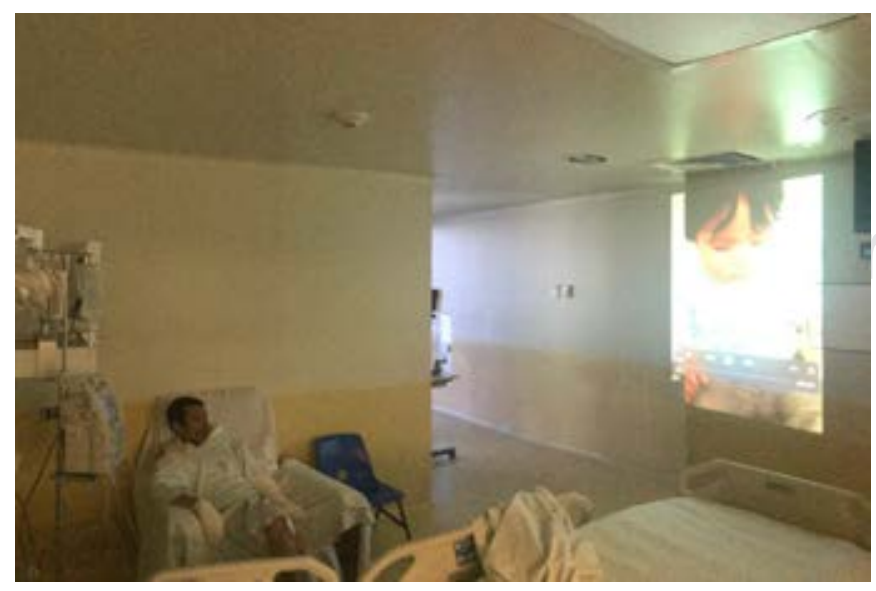

Figura 5: La terapia audiovisual representa una alternativa complementaria para disminuir el nivel de ansiedad en pacientes quemados.
La evaluación del nivel de ansiedad se realizó con la escala de Beck, el BDI (por sus siglas en inglés) ${ }^{33,34}$ y la escala hospitalaria de ansiedad y depresión (HOAD) antes y después de la proyección. ${ }^{35-38}$

La proyección de videos se realizó dos veces al día por el tiempo que el paciente permaneció en la unidad de cuidados intensivos en pacientes conscientes y sin delirio y concluyó hasta su egreso a piso. Estos video fueron solicitados por el personal médico a familiares con apoyo de una previa evaluación de psicología para hacer énfasis en cuál sería el personaje en cuestión que podría incentivar mejora al paciente.

Los videos incluyeron hijos, nietos, fiestas de cumpleaños, reuniones familiares, mensajes de amigos, entre otros.

Con esta estrategia se logró disminuir la ansiedad, además de que el paciente se presentaba más cooperador con el personal y aumentaban sus ganas de continuar con el tratamiento médico y de rehabilitación.

\section{CONCLUSIONES}

La ansiedad es una patología que se presenta con frecuencia en los pacientes en la unidad de cuidados intensivos, es de origen multifactorial y se relaciona directamente con la evolución de éstos y de su estancia hospitalaria.

La presente propuesta pretende implementar la terapia audiovisual como un método para disminuir el estado de ansiedad en los pacientes quemados, ya que este grupo de pacientes pasa por una estancia intrahospitalaria prolongada, además de múltiples factores psicológicos involucrados, y tiene la finalidad de hacer menos difícil el tratamiento del paciente.

No se han encontrado estudios similares al presente y es por ello que resulta importante implementar este tipo de material y realizar estudios con diferentes materiales audiovisuales en diferentes momentos de la estancia hospitalaria, además de ser una estrategia que puede utilizarse en diversos escenarios clínicos como delirium, depresión, síndrome de supresión, etcétera.

\section{REFERENCIAS}

Allué BM. Características psicológicas del gran quemado [Tesis]. España: Universidad de Oviedo; 2014.

2. Tedstone JE, Tarrier N. An investigation of the prevalence of psychological morbidity in burn-injured patients. Burns. 1997;23(7-8):550-554.

3. Gallach-Solano E, Pérez del Caz MD, Vivó-Benlloch C. Perfil psicológico del paciente gran quemado: prevalencia psicopatológica y variables asociadas. Cir Plast Iberolatinoam. 2015;41(4):427-436.

4. Zapata DM, Estrada A. Calidad de Vida relacionada con la salud de las personas afectadas por quemaduras después de la hospitalización. Biomédica. 2010;30(4):492-500.

5. Organización Mundial de la Salud. 2008. Disponible en: www. who.int/mediacentre/news/releases/2008/pr46/es/index.htlm. 
6. Wisely JA, Wilson E, Duncan RT, Tarrier N. Pre-existing psychiatric disorders, psychological reactions to stress and the recovery of burn survivors. Burns. 2010;36(2):183-191.

7. Giannoni-Pastor A, Goma-i-Freixanet M, Valero S, Fidel Kinori SG, Tasqué-Cebrián R, Arguello JM, et al. Personality as a predictor of depression symptoms in burn patients: a follow-up study. Burns. 2015;41(1):25-32.

8. Bond S, Gourlay C, Desjardins A, Bodson-Clermont P, Boucher ME. Anxiety, depression and PTSD-related symptoms in spouses and close relatives of burn survivors: when the supporter needs to be supported. Burns. 2017;43(3):592-601.

9. Fidel-Kinori SG, Eiroa-Orosa FJ, Giannoni-Pastor A, TasquéCebrián R, Arguello JM, Casas M. The Fenix II study: A longitudinal study of psychopathology among burn patients. Burns. 2016;42(6):1201-1211.

10. Vera I, Ferrando E, Vidal I, Gómez-Olmeda D, González JC. Accidentes por quemadura y enfermedad mental. Rev Psiquiatr Salud Ment (Barc.). 2010;3(1):19-22.

11. Carney BC, McKesey JP, Rosenthal DS, Shupp JW. Treatment strategies for hypopigmentation in the context of burn hypertrophic scars. Plast Reconstr Surg Glob Open. 2018;6(1):e1642.

12. Costas González M, Robles V, Iglesias JMC. Ansiedad y depresión entre los pacientes hospitalizados en el complejo hospitalario de Ferrol. Cuadernos de Medicina Psicosomática y Psiquiatría de Enlace. 2013;107:3.

13. Waqas A, Raza N, Zahid T, Rehman A, Hamid T, Hanif A, et al. Predictors of post-traumatic stress disorder among burn patients in Pakistan: the role of reconstructive surgery in postburn psychosocial adjustment. Burns. 2018;44(3):620-625.

14. Corry NH, Klick B, Fauerbach JA. Posttraumatic stress disorder and pain impact functioning and disability after major burn injury. J Burn Care Res. 2010;31(1):13-25.

15. Benaim F. Enfoque global del tratamiento de las quemaduras. En: Coiffman F (Ed.). Cirugía plástica reconstructiva y estética. Barcelona, Masson-Salvat; 1994. pp. 443-496.

16. Dalh O. Memories of pain, adaptation to life and early identification of stressors in the patients with burns [Theses]. Suecia: Karolinska Institutet; 2013.

17. Thomas BD, Ford CG, Addicks SH, Williford DN, Manegold EM, Randall CL, et al. Implementation of a psychosocial screener for adults in an outpatient burn clinic. J Burn Care Res. 2019;40(3):331-335.

18. Palmu R, Suominen K, Vuola J, Isometsa E. Mental disorders after burn injury: a prospective study. Burns. 2011;37(4):601-609.

19. Ullrich PM, Askay SW, Patterson DR. Pain, depression, and physical functioning following burn injury. Rehabil Psychol. 2009;54(2):211-216.

20. Weinberg K, Birdsall C, Vail D, Marano MA, Petrone SJ, Mansour EH. Pain and anxiety with burn dressing changes: patient self-report. J Burn Care Rehabil. 2000;21(2):155-156; discussion 157-161.

21. Palmu R, Suominen K, Vuola J, Isometsa E. Mental disorders among acute burn patients. Burns. 2010;36(7):1072-1079.

22. Davydow DS, Katon WJ, Zatzick DF. Psychiatric morbidity and functional impairments in survivors of burns, traumatic injuries, and ICU stays for other critical illnesses: a review of the literature. Int Rev Psychiatry. 2009;21(6):531-538.

23. Matlock KA, Yayah Jones NH, Corathers SD, Kichler JC. Clinical and psychosocial factors associated with suicidal ideation in adolescents with type 1 diabetes. $J$ Adolesc Health. 2017;61(4):471-477.

24. Griggs C, Goverman J, Bittner EA, Levi B. Sedation and pain management in burn patients. Clin Plast Surg. 2017;44(3):535-540.
25. Carrougher GJ, Ptacek JT, Honari S, Schmidt AE, Tininenko JR, Gibran NS, et al. Self-reports of anxiety in burn-injured hospitalized adults during routine wound care. J Burn Care Res. 2006;27(5):676-681.

26. Li J, Zhou L, Wang Y. The effects of music intervention on burn patients during treatment procedures: a systematic review and meta-analysis of randomized controlled trials. BMC Complement Altern Med. 2017;17(1):158.

27. Casasempere A, Enriquez I, Gallach E, Vivó C. Resultados funcionales y de calidad de vida a corto plazo en el paciente gran quemado. Poster Presentado al II Symposium Internacional Gran Quemado Getafe. 2014.

28. Seyyed-Rasooli A, Salehi F, Mohammadpoorasl A, Goljaryan S, Seyyedi Z, Thomson B. Comparing the effects of aromatherapy massage and inhalation aromatherapy on anxiety and pain in burn patients: a single-blind randomized clinical trial. Burns. 2016;42(8):1774-1780.

29. Choi J, Lee JA, Alimoradi Z, Lee MS. Aromatherapy for the relief of symptoms in burn patients: A systematic review of randomized controlled trials. Burns. 2018;44(6):1395-1402.

30. Hsu KC, Chen LF, Hsiep PH. Effect of music intervention on burn patients' pain and anxiety during dressing changes. Burns. 2016;42(8):1789-1796.

31. Najafi Ghezeljeh T, Mohades Ardebili F, Rafii F. The effects of massage and music on pain, anxiety and relaxation in burn patients: Randomized controlled clinical trial. Burns. 2017;43(5):1034-1043.

32. Konstantatos AH, Angliss M, Costello V, Cleland H, Stafrace S. Predicting the effectiveness of virtual reality relaxation on pain and anxiety when added to PCA morphine in patients having burns dressings changes. Burns. 2009;35(4):491-499.

33. Bradt J, Dileo C, Magill L, Teague A. Music interventions for improving psychological and physical outcomes in cancer patients. Cochrane Database Syst Rev. 2016;(8):CD006911.

34. Terol-Cantero MC, Cabrera-Perona V, Martín-Aragón M. Revisión de estudios de la Escala de Ansiedad y Depresión Hospitalaria (HAD) en muestras españolas. Anal Psicol. 2015;31(2):494-503.

35. Villegas P. Reporte de experiencia profesional [Tesis]. México: Facultad de Psicología, UNAM; 2004.

36. Rojas GC. Evaluar la validez de la escala de depresión geriátrica (GDS) y la escala de ansiedad y depresión para hospitales generales (HADS) [Tesis]. México: Facultad de Medicina, UNAM; 1991.

37. Whaley JA. Diagnóstico de ansiedad y depresión en pacientes quemados (escala HAD como una herramienta diagnóstica) [Tesis]. México: Facultad de Medicina, UNAM; 1992.

38. López JC, Verónica V, Martínez A, Sierra AE, et al. Exactitud y utilidad diagnóstica del Hospital Anxiety and Depression scale (HAD) en una muestra de sujetos obesos mexicanos. Rev Invest Clin. 2002;54(5):403-409.

\section{Correspondencia:}

Dr. Marco Antonio Garnica Escamilla

Calzada México Xochimilco Núm. 289,

Col. Arenal de Guadalupe Tepepan, 14389,

Alcaldía Tlalpan, CDMX.

Tel: 55 5999-1000, Ext. 14601

E-mail: teranestmarco@yahoo.com.mx 